\title{
INFLUENCIA DE LA LISTERIOSIS EN LA FERTILIDAD Y PRESENTACIÓN DE MASTITIS SUBCLÍNICA EN UN CONGLOMERADO LECHERO DE LA SABANA DE BOGOTÁ, COLOMBIA
}

\section{LISTERIOSIS INFLUENCE ON FERTILITY AND OCCURRENCE OF SUBCLINICAL MASTITIS IN A DAIRY CLUSTER OF SAVANNAH BOGOTA, COLOMBIA}

\author{
Manuel Isaac Gallego ${ }^{1}$, José Luis Azumendi ${ }^{2}$, Andrés Felipe Salazar ${ }^{3}$, Carolina Gallego ${ }^{4}$
}

\begin{abstract}
${ }^{1}$ Médico Veterinario, MSc., Docente Facultad de Ciencias Pecuarias, Programa de Medicina Veterinaria. Universidad de Ciencias Aplicadas y Ambientales U.D.C.A, calle 222 No 54-37, Bogotá, Colombia, e-mail: mgallego@udca.edu.co; ${ }^{2}$ Médico Veterinario, Director Científico. Fundación FUNCEP, calle 137 No. 12-15, Bogotá, Colombia, e-mail: funcepdircientifico@cable.net.co; ${ }^{3}$ Médico Veterinario. Ejercicio particular, calle 145 No. 21-61 Apto. 301B, Bogotá, Colombia, e-mail: asalazarmv@gmail.com; ${ }^{4}$ Médico Veterinario, MSc., c.PhD., Docente Facultad de Ciencias Pecuarias, Programa de Medicina Veterinaria. Universidad de Ciencias Aplicadas y Ambientales U.D.C.A, calle 222 No. 54-37, Bogotá, Colombia, e-mail: cgallego@udca.edu.co
\end{abstract}

Rev. U.D.C.A Act. \& Div. Cient. 18(2): 409-415, Julio-Diciembre, 2015

\section{RESUMEN}

Esta investigación tuvo como objetivo determinar la influencia de la listeriosis sobre el desempeño reproductivo y la presentación de mastitis subclínica, en un conglomerado lechero, de la Sabana de Bogotá. Se tomaron al azar ochenta y cuatro vacas en producción y en capacidad reproductiva, que fueron examinadas dos veces, con un intervalo de dos meses, para un total de 168 muestras, mediante la técnica de ELISA, para $\mathrm{IF}_{\gamma}$ bovino específico para listeriosis. Los resultados indicaron una incidencia de $36 \%$ de positividad a la enfermedad. Las vacas positivas a $\mathrm{IF}_{\gamma}$ presentaron mastitis subclínica grado $1(\mathrm{P}<0.00001)$ y grado $2(\mathrm{P}<0.3402)$; adicionalmente, las cifras de abortos en estas vacas fueron mayores $(\mathrm{P}<0.0002)$. En consecuencia, tuvieron mayor número de días abiertos $(\mathrm{P}<0.00001)$ y un número mayor de servicios por concepción $(\mathrm{P}<0.0093)$. Los resultados obtenidos ponen en evidencia los efectos negativos de la infección por Listeria monocytogenes sobre el tracto reproductivo y la responsabilidad del microorganismo en la presentación de mastitis en bovinos infectados.

Palabras clave: Infertilidad, mastitis, ganado de leche.

\section{SUMMARY}

This research aimed to determine the influence of listeriosis on the reproductive performance and the presentation of mastitis in a dairy cluster of the Sabana de Bogotá. We randomly selected eighty four cows in production and reproductive capacity in four farms, which were examined twice with an interval of two months for a total of 168 samples by ELISA test for specific bovine $\mathrm{IF}_{\gamma}$ for listeriosis. The results indicated an incidence of $36 \%$ of positivity to the disease. Positive cows for $\mathrm{IF}_{\gamma}$ had grade 1 of subclinical mastitis $(P<0.00001)$ and grade $2(P<0.3402)$. The figures for abortions in these animals were higher than negatives $(P$ $<0.0002)$. Accordingly, had higher number of open days ( $P$ $<0.00001)$ and a greater number of services per conception $(P<0.0093)$. The results highlight the negative influence of Listeria monocytogenes infection on the reproductive tract and responsibility of the microorganism in the presentation of mastitis in infected cattle.

Key words: Infertility, mastitis, dairy cattle.

\section{INTRODUCCIÓN}

La listeriosis ha estado involucrada como una entidad infecciosa de gran importancia para la salud de los seres humanos, al consumir alimentos contaminados, procedentes de animales infectados o del medio ambiente, convirtiéndose en un significativo contaminante, en todas las etapas de la cadena alimenticia (Tienungoon et al. 2000; Vanegas et al. 2007; Pérez-Trallero et al. 2014).

En los seres humanos induce el desarrollo de patologías, como abortos, anormalidades fetales, pérdidas neonatales, 
septicemia y diversos problemas nerviosos. En las mujeres, la infección materna es frecuentemente asintomática, mientras que la infección fetal y neonatal es severa y de pronóstico reservado, con 25-35\% de mortalidad (Charlier-Woerther \& Lecuit, 2014). La enfermedad en los bovinos cursa también con la presentación de lesiones en el sistema nervioso central, pero ha sido implicada también en problemas de tipo reproductivo, como pérdidas gestacionales y mortalidad perinatal, además de retención de placenta, metritis y mastitis (Czuprynski et al. 2010), con la consiguiente disminución de la fertilidad. Tanto en los seres humanos como en los animales, en general, la listeriosis está más referenciada como una enfermedad de tipo nervioso y reproductivo, ya que la bacteria puede cruzar las barreras placentaria, intestinal y nerviosa (Charlier-Woerther \& Lecuit, 2014); sin embargo, desde el punto de vista veterinario, no se han hecho estudios de su influencia sobre los diferentes parámetros reproductivos o productivos en ganado de leche.

La colonización del útero grávido y del feto ha sido reproducida experimentalmente por inoculación intravenosa, oral y respiratoria de animales gestantes de diferentes especies, como bovinos, ovinos, conejos, ratas, ratones y, en ellos, se ha demostrado que Listeria monocytogenes accede al feto por penetración hematógena de la barrera placentaria, lo cual, explica el tropismo especial que presenta el microorganismo por el útero grávido. La depresión local del sistema inmune en la placenta contribuye al incremento de la susceptibilidad a la infección por la bacteria. Lo anterior, se relaciona con la deficiencia en producción de Interferón-gamma $\left(\mathrm{IF}_{\gamma}\right)$, que facilita la invasión listerial de la placenta y de los tejidos fetales (Torres et al. 2005).

El diagnóstico de esta enfermedad ha descansado, usualmente, en el aislamiento bacteriológico, con el inconveniente que esta técnica no presenta la sensibilidad y la especificidad adecuadas, además de la demora en obtener los resultados; debido a lo anterior, se han diseñado diferentes técnicas de diagnóstico, como ELISA y PCR (Sierra et al. 2005; Vanegas et al. 2007). La técnica de $\left(\mathrm{IF}_{\gamma}\right)$ ha sido ampliamente usada en el diagnóstico de patógenos con ciclos de vida intracelulares, como Mycobacterium tuberculosis (Cho, 2007; Dheda et al. 2007), demostrando, en el caso de la listeriosis, ser altamente significativa $(P<0,01)$ con el aislamiento microbiológico (Gallego et al. 2015), lo cual, permite determinar, con seguridad, las posibles consecuencias de la enfermedad en el comportamiento reproductivo y productivo de los animales, a nivel de campo.

En Colombia, la bacteria ha sido reportada en casos humanos de meningoencefalitis (Sánchez et al. 1992; Crespo et al. 1999) y en alimentos de diferente naturaleza, como leches crudas (Díaz \& Muñoz, 1994) y quesos frescos (Muñoz \& Díaz, 1996), lo mismo que en productos cárnicos (Vanegas et al. 2007).
Este microorganismo fue detectado por Gallego et al. (2003), en un conglomerado lechero de la Sabana de Bogotá, en porcentajes que fluctuaron entre el 2,4 y $24 \%$ y Moreno et al. (2007), en un estudio sobre la contaminación microbiológica y la calidad de la leche realizado en el departamento de Boyacá, registraron que de 28 fincas, el 92,8\% presentó contaminación con $L$. monocytogenes. Posteriormente, Gallego et al. (2007) reportaron la presencia de la bacteria en canales de ganado Holstein, sacrificado en una planta de beneficio de la Sabana de Bogotá, en 2,26\%, de los canales examinados y, en canales de ganado cebú, un grado de contaminación del 8,33\%, con un rango entre el 3,4 y el 13,2\% (Villamil et al. 2007). Otros estudios adelantados por Gallego et al. (2015) hallaron una prevalencia, empleando la técnica de $\mathrm{IF}_{\gamma}$, del $31,36 \%$, en el mismo grupo de fincas de la Sabana de Bogotá, que fue empleado en la realización del presente trabajo.

La listeriosis es una enfermedad infecciosa que ha cobrado gran importancia en diferentes partes del mundo, especialmente, en la población bovina y ovina; en ambas especies, la enfermedad se puede manifestar como una infección del sistema nervioso, meningoencefalitis en adultos y meningitis en animales jóvenes, aborto, septicemia generalizada, afectando el hígado y otros órganos y mastitis (Czuprynski et al. 2010).

Gray \& Killinger (1966) mencionaron que L. monocytognes puede tener predilección por invadir el feto y la placenta de varios mamíferos. El aborto, se presenta, según estos autores, en el tercer trimestre de la gestación, con los signos clínicos obvios del problema, pudiendo ocurrir una presentación simultánea con problemas nerviosos, como se ha observado en ovejas (Vásquez-Boland et al. 1992), pudiendo ser detectada en los fetos abortados o placenta de los animales afectados.

De acuerdo con Barzoza-Lewis et al. (2012), el Interferón tipo I (I-IFN, IFN $\alpha \beta$ ) es la llave de la respuesta inmune a los patógenos virales; sin embargo, su papel frente a las infecciones bacterianas se está empezando a entender. Teniendo en cuenta estos estudios, se deja en evidencia un mecanismo mediante, el cual, el interferón tipo 1 regula la respuesta inmune a la listeria, a través de una regulación negativa de quimioquinas, conducentes al reclutamiento de neutrófilos.

Rayamaghi et al. (2010) comprobaron que la producción del interferón tipo I incrementa la susceptibilidad a L. monocytogenes, mientras que el tipo II o IFN- $-\gamma$, activa la resistencia de los macrófagos. Los autores hallaron que estos aspectos inmunológicos, se realizan por interrelaciones entre las respectivas vías de señalización, lo que permite la priorización del tipo de respuesta inmune y contribuye a los efectos benéficos del interferón. 
Uno de los aspectos más importante de la listeriosis es la implicación en la presentación de mastitis subclínica, lo cual, ha sido reportado por diversos autores que evaluaron el problema, mediante la inoculación experimental de la glándula mamaria (Gitter et al. 1980; Czuprynski et al. 1989; Fthenakis et al. 1998; Tzora et al. 1998; Maliki et al. 2002; Winter et al. 2004; Czuprynski et al. 2010). Estudios presentados por Jamali \& Radmehr (2013) mostraron que los serogrupos que producen mastitis son, principalmente, $4 \mathrm{~b}, 4 \mathrm{~d}, 4 \mathrm{e}, 1 / 2 \mathrm{a}$, 3a, 1/2b, 3b, 7, 1/2c, 3e todos codificados en inlA, inlC, inlG, necesarios para la internalización de Listeria spp. en células intestinales y empleados para la diferenciación de cepas virulentas y avirulentas.

Los autores anteriores coinciden en que $L$. monocytogenes puede ser detectada en la leche por períodos variables, incluso, en cantidades pequeñas; por lo tanto, es muy posible que las vacas positivas -en la técnica de ELISA para $\mathrm{IF}_{\gamma} \mathrm{y}$ que presenten reacción positiva en CMT-, uno de las posibles microorganismos involucrados sea L. monocytogenes, dada la sensibilidad de la técnica y su correlación con los cultivos bacteriológicos. Considerable atención ha tenido la presentación de la mastitis, que puede tener varios grados de severidad, desde una forma subclínica a una infección supurativa severa. Gitter et al. (1980), Doyle et al. (1987) y Czuprynski et al. (2010) establecieron que $L$. monocytogenes pudo ser encontrada en los neutrófilos en leche de cuatro vacas examinadas, que presentaron mastitis. La infección prolongada de la ubre puede ocasionar incremento de eliminación de la bacteria, especialmente, en períodos de inmunodepresión. Chen \& Novick (2009) hallaron una asociación estrecha entre $L$. monocytogenes y $S$. aureus, que puede tener un gran significado en la presentación de mastitis por listeriosis.

Las cifras anteriores revelan la importancia que pueda tener la enfermedad desde el punto de vista de salud pública, como un contaminante de alimentos de origen animal; sin embargo, hasta el momento no se ha realizado una investigación que ponga de manifiesto el posible impacto de la enfermedad sobre la fertilidad y la productividad en los hatos lecheros.

\section{MATERIALES Y MÉTODOS}

De acuerdo con la Asociación de Ganaderos de Facatativá (Castro, 2013), en una población de 21.631 bovinos del conglomerado lechero de los municipios de Madrid, Mosquera y El Rosal, donde se registró una prevalencia de $24 \%$ de positividad, mediante exámenes bacteriológicos (Gallego et al. 2003), se seleccionaron 84 bovinos, en capacidad reproductiva y en producción, ubicados en cuatro fincas, de acuerdo a una frecuencia esperada del $36 \%$ y un nivel de confianza del 99\% (Kish, 1972; Kuehl, 2001). Las fincas fueron seleccionadas de acuerdo a los siguientes criterios: explotaciones lecheras, atendidas directamente por sus propietarios; con predominio de la raza Holstein; asistencia veterinaria; registros de producción y de reproducción; certificadas libres de brucelosis; con planes de inseminación artificial; control de enfermedades parasitarias e infecciosas, especialmente, del tracto reproductivo y mastitis. No se tuvieron en cuenta fincas negativas a listeriosis, dadas las prevalencias altas de la enfermedad, encontradas anteriormente en este conglomerado lechero y que $L$. monocytogenes es considerado un microorganismo ambiental (Orndorff et al. 2006).

De las vacas seleccionadas al azar, se tomaron dos muestras de sangre con heparina, con intervalos de dos meses, para un total de 168 muestras; fueron transportadas al laboratorio en refrigeración, en un período de cinco horas. A las muestras seleccionadas, se les realizó cuantificación de $\mathrm{IF}_{\gamma}$, como indicador de la inmunidad celular para L. monocytogenes, de acuerdo al protocolo establecido por Gallego et al. (2015).

En el momento de la toma de muestras de sangre a las vacas en producción, se les realizó la prueba de California Mastitis Test (CMT), de acuerdo al protocolo e interpretación de Schalm \& Nordlander (1957).

Con el fin de analizar la influencia de la enfermedad sobre el comportamiento reproductivo, se tomaron -de los registros de las fincas, en el momento de la visita-, la información relacionada con los parámetros reproductivos, de acuerdo con Gallego (2009). A la información obtenida, se le realizó una estadística descriptiva y se analizó la significancia, mediante las técnicas de Chi cuadrado, de acuerdo al programa EPIINFO (2014).

Conforme con los resultados, se formaron dos grupos de vacas, tomando, en uno de ellos, los animales con días abiertos, superiores a $100 \mathrm{y}$, del otro, los que presentaron cifras menores de 100. Para el intervalo entre partos, se tuvo en cuenta el rango de vacas, con un número igual o menor de 380 días y mayor, de 380 . En relación con los servicios por concepción, se fijó como parámetro, animales con un servicio por concepción y los que presentaron dos o más servicios. En mastitis, se formaron, de la misma manera, grupos positivos y negativos a la enfermedad y se evaluó cada uno de los cuartos en las ubres, de acuerdo a la escala de Schalm \& Nordlander (1957).

En los dos grupos de vacas, positivos y negativos a la prueba de $\mathrm{IF}_{\gamma}$, se compararon los parámetros encontrados, tanto superiores como inferiores, de las variables consideradas como normales. 


\section{RESULTADOS Y DISCUSIÓN}

Todos los predios involucrados en este estudio disponían de asistencia profesional y eran atendidos directamente por sus propietarios. En estos predios, no se hallaron evidencias anteriores o actuales de problemas generales de salud o relacionados con factores nutricionales, enfermedades infecciosas y parasitarias o de manejo, lo que permite descartar la influencia de estos aspectos, en los resultados obtenidos.

Los primeros estudios sobre la enfermedad en el mismo conglomerado de leche y bajo las mismas condiciones reportaron una prevalencia máxima del $24 \%$, mediante exámenes bacteriológicos (Gallego et al. 2003). En la presente investigación, mediante el empleo de una técnica de mayor sensibilidad y especificidad, la prevalencia encontrada fue del $36 \%$, en las dos mediciones que se efectuaron con dos meses de intervalo, por lo tanto, es deducible que la técnica de $\mathrm{IF}_{\gamma}$ presenta una mayor utilidad, en la detección de animales positivos a listeriosis.

En relación con el grado de reactividad a la prueba de CMT, del total de animales examinados, 16 (9,52\%) fueron positivos. Al realizar la prueba de ELISA para $\mathrm{IF}_{\gamma}$, se evidenció una relación estadística significativa en las vacas que presentaron grado uno $(\mathrm{P}<0,0001)$ y grado dos $(\mathrm{P}<0,009)$, consideradas como subclínicas, lo cual, está relacionado con los hallazgos presentados por Czuprynski et al. (1989), Bourry et al. (1995), Fthenakis et al. (1998), Tzora et al. (1998) y Winter et al. (2004). Las mastitis tipo 3 y 4 no fueron evaluadas, debido al escaso número de animales que presentaron esta condición.

El hallazgo de L. mpnocytogenes en la leche y la presentación de mastitis tendrían como origen la contaminación bacteriana suscitada de la eliminación en las materias fecales o como consecuencia de la septicemia y necrosis focal en hígado y en bazo, de los de los animales infectados. El desarrollo de la mastitis puede variar desde una forma subclínica a supurativa con localización de la bacteria en los neutrófilos y la consecuente reactividad de la prueba de CMT. La infección que se establece a largo plazo en la leche, acompañada de la eliminación de la bacteria en la leche, puede ser exacerbada periódicamente por inmunodepresión, debida a enfermedades subyacentes u otras causas. Este fenómeno tiene implicaciones prácticas, ya que el tratamiento de las mastitis por L. monocytogenes sería difícil de lograr (Czuprynski et al. 2010). Es importante tener en cuenta la posible asociación de este microorganismo con otros agentes, ya sea desde el punto de vista patológico o de protección, como el Staphylococcus aureus (Chen \& Novick, 2009).

La eliminación de Listeria monocytogenes, a través de la leche en vacas infectadas, constituye uno de los principales problemas en salud pública, debido a las implicaciones que presenta el organismo para la salud humana (Díaz \& Muñoz, 1994; Muñoz \& Díaz, 1996; Gallego et al. 2003; Moreno et al. 2007), ya que las vacas lecheras pueden ser reservorios de una población diversa de $L$. monocytogenes patógenas para humanos, con el consiguiente peligro en salud pública (Haley et al. 2015). El empleo de técnicas de diagnóstico de alta sensibilidad y especificidad, como la técnica de ELISA para $\mathrm{IF}_{\gamma}$, para la detección de animales positivos, permitiría el establecimiento de programas más eficientes de control de la enfermedad.

Al analizar el comportamiento reproductivo de los animales, se encontró, en relación con el número de días abiertos correspondientes al penúltimo parto, una diferencia significativa $(P<0,0001)$ en las positivas en la técnica de ELISA para $\mathrm{IF}_{\gamma}$, que presentaron un número de días abiertos superiores a 100, en comparación con los que mostraron cifras inferiores, a este parámetro.

Igualmente, en las vacas positivas a $\mathrm{IF}_{\gamma}$, que mostraron un incremento significativo en los días abiertos superiores a 100, se evidenció, además, un incremento significativo en el número de servicios superiores a uno $(P<0,009)$.

En relación con la presentación de abortos, en el presente estudio se halló una alta relación estadística $(P<0,0002)$, entre las vacas positivas a la prueba de ELISA para $\mathrm{IF}_{\gamma}$, con la presentación de uno o más abortos en su vida reproductiva, en comparación con las negativas que, en el momento del estudio, manifestaban un comportamiento reproductivo normal. Aunque no existe historia sobre los posibles agentes etiológicos o causas de los abortos presentados anteriormente, es evidente la significancia estadística, que existe entre la positividad a listeriosis y la exposición de abortos. Este factor, al mismo tiempo, incide en el incremento en el número de días abiertos y puede ser una de las causas del incremento, en los servicios por concepción.

La influencia de $L$. monocytogenes sobre el comportamiento reproductivo de las vacas ha sido estudiando por diferentes autores (Abram \& Doric, 1997; Parkash et al. 1998; Gulleria \& Pollard, 2000; Vásquez-Boland et al. 2001; Torres et al. 2005), por inoculación intravenosa, oral y respiratoria, en animales gestantes susceptibles, como ovejas, reses, conejos, cobayos y ratas. De esta manera, se demostró que la bacteria accede al feto por penetración hematógena.

Entre los principales mecanismos por los cuales L. monocytogenes puede desencadenar la presentación de aborto, Engeland et al. (1997) planteó una posible explicación de tipo hormonal. Después de la inoculación del microorganismo encontró una disminución significativa en los niveles de progesterona y un incremento en 15-ketodihydro-PGFF $\alpha$. 
Esta alteración en la función endocrina placentaria podría ser la responsable del aborto por la bacteria; plantearon, además, que durante la preñez se presenta un período de inmunodepresión, para evitar el rechazo allográfico del feto, un hecho que se puede manifestar ante cualquier enfermedad infecciosa y que no es exclusivo de listeriosis. A este fenómeno, se puede añadir el hecho que $L$. monocytogenes induce una disminución de la expresión de la hemo-oxigenasa-1 (HO-1) en las células del trofoblasto, importantes para reducir la expresión de las HO-1, lo cual, puede influir en la presentación del aborto, tal como fue observado por Irvin et al. (2008) en ratones.

Tachibana et al. (2011) descubrieron un aspecto adicional. El gen Bcl-XL, el cual, presenta un factor de protección placentaria en unión de las $\mathrm{HO}-1$, podría estar subregulado en placentas de ratones infectados con $L$. monocytogenes; por lo tanto, la bacteria induciría una muerte celular, por un mecanismo alterno dependiente de la $\mathrm{HO}-1$ y del gen Bcl-XL. Poulsen et al. (2011) compararon ratones genéticamente resistentes a la infección por $L$. monocytogenes con ratones susceptibles, a una inoculación intragástrica de diseminación del microorganismo a los órganos fetales, a los tres días de inoculación. Los primeros fueron más resistentes que los susceptibles, durante la preñez; la inflamación y la necrosis fueron más severas en los susceptibles.

La presentación del aborto por $L$. monocytogenes ha sido establecida en forma definitiva por diferentes autores, en varias especies; en la especie bovina, el mecanismo empleado por el microorganismo no ha sido dilucidado específicamente, por lo tanto, establecer la patogénesis del microorganismo en los bovinos contribuiría al desarrollo de programas de control de la enfermedad.

Financiación: Este estudio fue financiado por la Universidad de Ciencias Aplicadas y Ambientales U.D.C.A. Conflicto de intereses: El manuscrito fue preparado y revisado con la participación de todos los autores, quienes declaramos que no existe ningún conflicto de intereses que ponga en riesgo la validez de los resultados presentados.

\section{BIBLIOGRAFÍA}

1. ABRAM, M.; DORIC, M. 1997 Primary Listeria monocytogenes infection in gestating mice. Folia Microbiol. (Czech Republic). 42:65-71.

2. BARZOZA-LEWIS, K.L.; HOTH, J.J.; HILTBOLD, E.M. 2012. Type I interferon signalling regulates the composition of inflamatory infiltrates upon infectión with Listeria monocytogenes. Cellular Immunol. (USA). 73:41-51.3. BOURRY, A.; POUTREL, B.; ROCOURT,
J. 1995. Bovine mastitis caused by Listeria monocytogenes of natural and experimental infections. J. Med. Microbiol. (USA). 3:125-132.

3. CASTRO, I. 2013 Asociación de Ganaderos de Facatativá. Comunicación personal.

4. CHEN, J.; NOVICK, R.P. 2009. Phage-mediated intergeneric transfer of toxin genes. Science (USA). 323:139-141.

5. CHO, S. 2007. Current issues on molecular and immunological diagnosis of tuberculosis. Yonsei Medical J. (Japan). 48(3):347-359.

6. CRESPO, M.; VÉLEZ, J.; CASTAÑEDA, C.; HOYOS, F.; LÓPEZ, M.; SALAZAR, J. 1999. Aislamiento de Listeria monocytogenes en un hospital de tercer nivel. Col. Médica (Colombia). 30(2):89-98.

7. CHARLIER-WOERTHER, C.; LECUIT, M. 2014 Listeriosis and pregnancy. Presse Med. (France). 43(6):676682.

8. CZUPRYNSKI, C.J.; NOEL, E.J.; DOYLE, M.P.; SCHULTZ, R.D. 1989. Ingestion and killing of Listeria monocytogens by blood and milk phagocytes from mastitic and normal cattle. J. Clin. Microbiol. (USA) 27:812-817.

9. CZUPRYNSKI, C.J.; KATHARIOU, S.; POULSEN, K. 2010. Listeria. En: Gyles, C.L.; Prescott, J.F.; Songer, J.G.; Thoen, C.O. (eds). Pathogenesis of Bacterial Infections in Animals. Ed. Wiley-Blackwell (USA). p.167-187.

10. DHEDA, K.; POORAN, A.; PAI, M.; MILLER, R.; LESLEY, K.; BOOTH, H.; SCOTT, G.; AKBAR, A.; ZUMLA, A.; ROOK, G. 2007. Interpretation of Mycobacterium tuberculosis antigen-specific IFNy release assays (T-SPOT.TB) and factors that may modulate test results. J. Infection. (England). 5:169-173.

11. DÍAZ, G.; MUÑOZ, A. 1994. Incidencia de Listeria monocytogenes en leches crudas y leches pasteurizadas en el altiplano Cundiboyacense. Biomédica (Colombia) 4:58.

12. DOYLE, M.P.; GLASS, J.T.; BEERY, J.T.; GARCIA, G.A.; POLLARD, D.J.; SCHULTZ, R.D. 1987. Survival of Listeria monocytogenes in milk during high temperature, short time of pasteurization. Applied Envir. Micro. (USA). 53:1433-1438. 
13. ENGELAND, I.V.; WALDELAND, H.; RAPSTAEL, E.; KINDAHL, H.; ANDRESEN, O. 1997. Effect of experimental infection with Listeria monocytogenes on the development of pregnancy and concentration of progesterone, oestrone sulphate and 15-ketodihydroPGF2 $\alpha$ in the goat. An. Reprod. Sci. (USA). 5:311327.

14. EPI-INFO ${ }^{\mathrm{rm}}$ 3.5.4. 2014. Database and Static Software; Public Health Profesional org.; Center for Disease control and Prevention. Disponible desde internet en: www.cdc.gov/epiinfo/7/ (con acceso 13/10/2014).

15. FTHENAKIS, G.C.; SARATSIS, P.H.; TZORA, A.; LINDE, K. 1998. Naturally occurring suclinical ovine mastitis associated by Listeria monocytogenes. Small Ruminant. Res. (USA). 31:23-27.

16. GALLEGO, M.I.; TORRES, O.A.; SOTO, C.Y.; DUQUE, D.C.; BENÍTEZ, C. 2003. Determinación de portadores de Listeria sp. en un conglomerado lechero de la vereda Puente de Piedra municipio de Madrid (Cundinamarca, Colombia). Rev. U.D.C.A Act. \& Div. Cient. (Colombia). 6(1):49-56.

17. GALLEGO, M.I.; MANRIQUE, P.C.; TORRES, O.A.; RAMÍREZ, M.F. 2007. Listeria monocytogenes en canales de ganado Holstein en una planta de sacrificio de la Sabana de Bogotá (Colombia). Rev. U.D.C.A. Act. \& Div. Cient. 8(2):95-101.

18. GALLEGO, M.I. 2009 Manejo del problema reproductivo en ganado de leche. Universidad de Ciencias Aplicadas y Ambientales. Fondo de Publicaciones. Digiprint Editores Bogotá, Colombia. 232p.

19. GALLEGO, L.C.; GALLEGO, M.I.; AZUMENDI, J.L.; PAIPA, J.; JARAMILLO, D. 2015. Estandarización de la técnica de Interferón-gamma (IFN-Y) para la detección de Bovinos infectado con Listeria monocytogenes. Rev. U.D.C.A Act. \& Div. Cient. 18(1):163-170.

20. GITTER, M.; BRADLEY, R.; BLAMPIED, P.H. 1980. Listeria monocytogenes infection in bovine mastitis. Vet. Record. (England). 107:390-393.

21. GRAY, M.L.; KILLINGER, A.H. 1966. Listeria monocytogenes and listeric infections. Bacteriology Reviews (Canada). 30:309-382.

22. GULLERIA, I.; POLLARD, J.W. 2000. The trophoblast is a component of the innate immune system during pregnancy. Nature Medicine. (England). 6:589-593.
23. HALEY, B.J.; SONNIER, J.; SCHUKKEN, Y.H.; KARNS, J.S.; VAN KESSEL, J.A. 2015. Diversity of Listeria monocytogenes within a U.S dairy herd, 2004-2010. Food Borne Pathog. Dis. (USA). 10:844-850.

24. IRVIN, E.A.; WILLIAMS, D.; VOSS, K.A.; SMITH, M.A. 2008. Listeria monocytogenes infection in pregnant guinea pigs associated with maternal liver necrosis, a decrease in maternal TNFa concentration and increase in placental apoptosis. Reprod. Toxicol. (USA). 26:123-129.

25. JAMALI, H.; RADMEHR, B. 2013. Frequency, virulence, genes and antimicrobial resistance of Listeria sp. isolated from bovine clinical mastitis. Vet. J. (England). 198:541-542.

26. KISH, L. 1972. Muestreo de encuestas. Edit. Trillas. $1^{\circ}$ Ed. México D.F. 739p.

27. KUEHL, R.O. 2001 Diseño de experimentos. Ed. Thomson. México D.F. 666p.

28. MALIKI, S.V.S.; BARBUDDHE, S.B.; CHANDARI, S.P. 2002. Listeric infections in humans and animals in the Indian subcontinent. A review. Trop. Animal Health Prod. (England). 34(5):359-381.

29. MUÑOZ, A.; DÍAZ, G. 1996. Determinación e identificación de Listeria monocytogenes en quesos frescos y madurados que se comercializan en Santafé de Bogotá. Notinvima. (Colombia). 1:19-21.

30. MORENO, V.F.C.; RODRÍGUEZ, M.J.; MÉNDEZ, M.M.V.M.; OSUNA, A.L.E.; VARGAS, M.R. 2007. Análisis microbiológico y su relación con la calidad higiénica y sanitaria de la leche producida en la región del Alto de Chicamocha (departamento de Boyacá). Rev. Med. Vet. Univ. De la Salle. (Colombia). 14:61-83.

31. ORNDORFF, S.V.S.; HANRICK, T.S.; SMOAK, I.W.; HAVELL, E.A. 2006. GráfHost and bacterial factors in listeriosis pathogenesis. Vet. Microb. (USA). 114(12): $1-5$.

32. PARKASH, V.; MOROTTI, R.A.; JOSHI, V.; CARTUN, R.; RAUCH, C.A.; WEST, A.B. 1998. Immunohistochemical detection of Listeria antigen in the placenta in perinatal listeriosis. Int. J. Gynec. Pathol. (USA) 17:343-350.

33. PÉREZ-TRALLERO, E.; ZIGORRAGA, C.; ARTIEGA, J.; ALKORTA, M.; MARIMÓN, J.M. 2014. Two outbreaks 
of Listeria monocytogenes, northern Spain. Emerg. Inf. Dis. (USA). 20(12):2155-2157.

34. POULSEN, K.F.; FAITH, N.G.; STEINBERG, H.; CZUPRYNSKI, C.J. 2011. Pregnancy reduce the genetic resistance of C57BC/b mice to Listeria monocytogenes infection by intragastric inoculation. Microbial Pathogenesis (USA) 50:360-366.

35. RAYAMAGHI, M.; HUMANN, J.; PENHEITER, K.; ANDREASEN, K.; LENZ L. 2010. The induction of IFN- $\alpha \beta$ enables Listeria monocytogenes suppress macrophage activation by IFN- $\gamma$. J. Exp. Med. (USA). 207(2):327-337.

36. SÁNCHEZ, E.; PARDO, R.; DUQUE, A.; PALOMINO, S.; REYES, E. 1992. Listeriosis del sistema nervioso central. Formas meníngeas agudas supurativas y romboencefálica. Acta Neurol. (Colombia). (3):165168.

37. SCHALM, O.; NORDLANDER, D. 1957. Experiments and observations leading to the development of the California Mastitis Test. J. Am. Vet. Med. Assoc. (USA). 130:189-204.

38. SIERRA, S.C.; POUTOU, R.A.; CARRASCAL, A.K.; TORRES, K.J.; MERCADO, M. 2005. Validación de PCR para detección de Listeria monocytogenes en quesos frescos. Rev. U.D.C.A Act. \& Div. Cient. 7(2):5365.

39. TACHIBANA, M.; HASHIDO, M.; NISHIDA, T.; SHIMIZU, T.; WATARAI, M. 2011. Protective role of heme Oxygenase-1 in Listeria monocytogenes-induced abortion. PLoS ONE 6(9):e25046.doi:10.1371/journal.pone.002546.

40. TIENUNGOON, S.; RATOWSKY, D.; MCMEEKEN, T.; ROSS, T. 2000. Growth limits of Listeria monocytogenes as a function of temperature, $\mathrm{pH}$. $\mathrm{NaCl}$ anlominod lactic acid. Appl. Environm. Microbiol. (USA) 66(4):709-772.

41. TORRES, K.; SIERRA, S.; POUTOU, R.; CARRASCAL, A.; MERCADO, M. 2005. Patogenesis de Listeria monocytogenes. Microorganismo zoonótico emergente. Rev. MVZ-Córdoba (Colombia). 10(1):511543.

42. TZORA, A.; FTHENAKIS, G.C.; LINDE, K. 1998. The effects of inoculation of Listeria monocytogenes into the ovine mammary gland. Vet. Microbiol. (USA) 59:193-202.

43. VANEGAS, L.M.C.; MARTINEZ, L.A.J.; BOTINA, B.L. 2007. Detección por PCR de Listeria monocytogenes en productos cárnicos distribuidos en Bogotá. Rev. U.D.C.A Act. \& Div. Cient. 9(2):149-156.

44. VÁSQUEZ-BOLAND, J.; KUHN, M.; BERCHE, P.; CHAKRABORTY, T.; DOMÍNGUEZ-BERNAL, G.; GOEBEL, W.; GONZÁLEZ-ZORN, B.; WEHLAND, J.; KREFT, J. 2001 Listeria pathogenesis and molecular virulence determinants. Clin. Microbiol. Rev. (USA) 14(3):584-640.

45. VÁSQUEZ-BOLAND, J.A.; DOMINGUEZ, L.; BLANCO, M.; ROCOURT, J.; FERNANDEZ-GARAIZABAL, J.M.; GUTIÉRREZ, C.B.; TASCÓN, R.I.; RODRÍGUEZFERRI, E.F. 1992. Epidemiological investigations of a silage associated epizootic of ovine listeric encephalitis using a new Listeria-selective enumeration mediun and phage typing. Am. J. Vet. Res. (USA). 3:368-371.

46. VILLAMIL, D.; GALLEGO, M.M.I.; TORRES, O.A.; RAMÍRES, M.F. 2007. Listeria monocytogenes en canales de bovinos cebú en una planta de sacrificio de la Sabana de Bogotá (Colombia). Rev. U.D.C.A Act. \& Div. Cient. 10(1):93-101.

47. WINTER, P.; SCHILCHER, F.; BAGO, Z.; SCHOEDER, D.; EGERBACHER, M.; BAUMGARTN ER, W.; WAGNER, M. 2004. Clinical and histopathological aspects of naturally occurring mastitis caused by Listeria monocytogenes in cattle and ewes. J. Vet. Med. B. Infect. Dis. Vet. Public Health. (USA) 51(4):176-179.

Recibido: Diciembre 2 de 2014

Aceptado: Noviembre 12 de 2015

Cómo citar:

Gallego, M.I.; Azumendi, J.L.; Salazar, A.F.; Gallego, C. 2015. Influencia de la Listeriosis en la fertilidad y presentación de mastitis subclínica en un conglomerado lechero de la sabana de Bogotá. Colombia. Rev. U.D.C.A Act. \& Div. Cient. 18(2): 409-415. 